\title{
Genome-wide association of mood-incongruent psychotic bipolar disorder
}

\author{
FS Goes ${ }^{1,8}$, ML Hamshere ${ }^{2,8}$, F Seifuddin ${ }^{1}$, M Pirooznia ${ }^{1}$, P Belmonte-Mahon ${ }^{1}$, R Breuer ${ }^{3}$, T Schulze ${ }^{4}$, M Nöthen ${ }^{5}$, S Cichon $^{5}$, \\ M Rietschel ${ }^{3}$, P Holmans ${ }^{2}$, PP Zandi ${ }^{6}$, Bipolar Genome Study (BiGS), N Craddock ${ }^{2,9}$ and JB Potash ${ }^{7,9}$
}

Mood-incongruent psychotic features (MICP) are familial symptoms of bipolar disorder (BP) that also occur in schizophrenia (SZ), and may represent manifestations of shared etiology between the major psychoses. In this study we have analyzed three large samples of BP with imputed genome-wide association data and have performed a meta-analysis of 2196 cases with MICP and 8148 controls. We found several regions with suggestive evidence of association $\left(P<10^{-6}\right)$, although no marker met genome-wide significance criteria. The top associations were on chromosomes: 6 q14.2 within the PRSS35/SNAP91 gene complex (rs1171113, $P=9.67 \times 10^{-8}$ ); 3p22.2 downstream of TRANK/LBA1 (rs9834970, $P=9.71 \times 10^{-8}$ ); and 14q24.2 in an intron of NUMB (rs2333194, $P=7.03 \times 10^{-7}$ ). These associations were present in all three samples, and both rs 1171113 and rs2333194 were found to be overrepresented in an analysis of MICP cases compared with all other BP cases. To test the relationship of MICP with SZ, we performed polygenic analysis using the Psychiatric GWAS Consortium SZ results and found evidence of association between SZ polygenes and the presence of MICP in BP cases (meta-analysis $P=0.003$ ). In summary, our analysis of the MICP phenotype in BP has provided suggestive evidence for association of common variants in several genes expressed in the nervous system. The results of our polygenic analysis provides support for a modest degree of genetic overlap between BP with MICP and SZ, highlighting that phenotypic correlations across syndromes may be due to the influence of polygenic risk factors.

Translational Psychiatry (2012) 2, e180; doi:10.1038/tp.2012.106; published online 23 October 2012

\section{Introduction}

Bipolar disorder (BP) is among the more reliably diagnosed ${ }^{1}$ and most heritable illnesses in psychiatry. ${ }^{2}$ Although a recent large-scale genome-wide association study (GWAS) metaanalysis found strong evidence for association of common markers in the calcium channel subunit gene CACNAC1 and the cell-surface protein gene ODZ4 with BP, the effect sizes are modest and account for only a small proportion of the phenotypic variance and heritability. ${ }^{3}$ Several hypotheses have been proposed to explain why gene identification in psychiatric phenotypes such as BP has met with less success than might have been anticipated. Among these are a higher burden of rare variants, ${ }^{4}$ a more polygenic genetic architecture $^{5}$ and a more heterogeneous phenotype. ${ }^{6}$

If the search for etiology is complicated by phenotypic heterogeneity, a potential method to mitigate this problem might be to delineate clinical subphenotypes that could be more biologically homogenous. Such phenotypes may also help uncover potential susceptibility risk factors for domains of psychopathology that may be shared across diagnoses. In this study, we focus on the Diagnostic and Statistical Manual of Mental Disorders (DSM)-IV subtype of BP with moodincongruent psychotic features (MICP). This subphenotype was first described a century ago as a potential diagnostic tool to differentiate the more common mood-congruent psychotic symptoms of mood disorders from the mood-incongruent psychotic symptoms of SZ. ${ }^{7}$ More modern conceptions of BP no longer exclude cases with mood-incongruent psychotic symptoms, which are now believed to comprise one-third to one-half of all psychotic BP. ${ }^{8,9}$ Mood-incongruent psychotic symptoms in BP appear to be important markers of severity, being associated with worse overall outcome, ${ }^{10,11}$ poor lithium responsiveness $^{12}$ and higher rates of attempted suicide. ${ }^{8,9}$

The presence of MICP symptoms might also be a manifestation of overlapping genetic susceptibility between BP and SZ. Family studies have found a modestly elevated risk of $S Z$ in relatives of cases with mood disorders and MICP, ${ }^{13,14}$ and vice versa. ${ }^{15}$ More recently, two genome-wide linkage scans of BP with MICP have been performed; ${ }^{9,16}$

\footnotetext{
${ }^{1}$ Department of Psychiatry, Johns Hopkins University School of Medicine, Baltimore, MD, USA; ${ }^{2}$ Department of Psychological Medicine, MRC Centre for Neuropsychiatric Genetics and Genomics, School of Medicine, Cardiff University, Cardiff, UK; ${ }^{3}$ Department of Genetic Epidemiology in Psychiatry, Central Institute of Mental Health, Mannheim, Germany; ${ }^{4}$ Department of Psychiatry and Psychotherapy, University of Göttingen, Göttingen, Germany; ${ }^{5}$ Department of Genomics, Life \& Brain Center, University of Bonn, Bonn, Germany; ${ }^{6}$ Department of Mental Health, Johns Hopkins Bloomberg School of Public Health, Baltimore, MD, USA and ${ }^{7}$ Department of Psychiatry, Carver College of Medicine, University of lowa, lowa City, IA, USA

Correspondence: Dr FS Goes, Department of Psychiatry, Johns Hopkins University School of Medicine, Meyer 4-119A, 600 N. Wolfe Street, Baltimore, MD 21287, USA. E-mail: fgoes1@jhmi.edu or Professor N Craddock, Department of Psychological Medicine, MRC Centre for Neuropsychiatric Genetics and Genomics, School of Medicine, Cardiff University, Heath Park, Cardiff CF14 4XN, UK.

E-mail: craddockn@ cardiff.ac.uk

${ }^{8}$ These authors contributed equally to this work.

${ }^{9}$ These authors contributed equally to this work.

Keywords: bipolar disorder; genome-wide association study; psychosis; schizophrenia; SNAP91; TRANK1

Received 6 September 2012; accepted 6 September 2012
} 
while there was limited overlap between the two, a number of genome-wide suggestive signals emerged in regions previously implicated in SZ linkage studies, including on chromosomes 1p32, 2q12 and 13q31.

We hypothesize that the BP subtype with MICP features may help uncover susceptibility genes for BP not readily detectable by examining the broad BP phenotype. Given the clinical similarities between MICP symptoms in BP and in SZ, we also hypothesize that some of these susceptibility genes would show partial overlap with susceptibility genes for SZ. To test these hypotheses, we have performed a GWAS analyses of the MICP phenotype using several large BP data sets and further sought to determine whether our findings might also influence susceptibility to SZ, by making use of the recently published Psychiatric GWAS Consortium (PGC) SZ results.

\section{Materials and methods}

Subjects. Subjects with BP and MICP were initially drawn from three large, independent samples of $\mathrm{BP}$, here termed the National Institute of Mental Health Bipolar Disorder Consortium (NIMH), the Wellcome Trust Case-Control Consortium (WTCCC) and the German samples. Subject collection and genotyping were conducted separately for each sample. However, quality control steps taken in the analyses of these samples have been identical.

NIMH sample. Eligibility, ascertainment and assessment procedures for the NIMH sample have been previously described. ${ }^{17}$ All subjects were assessed with the Diagnostic Interview for Genetic Studies (DIGS), and this was combined with family informant data and medical records to assign diagnoses based on DSM-III-R or DSM-IV criteria. Controls were ascertained throughout the United States and were genotyped as part of the Molecular Genetics of Schizophrenia II (MGS2) Collaboration. ${ }^{18}$ All control subjects completed a psychiatric questionnaire and those endorsing a history of BP, psychosis or symptoms sufficient for a diagnosis of major depression were excluded. Controls were matched to cases according to ethnicity and sex.

Wellcome Trust Case-Control Consortium. The WTCCC sample comprised the original 1868 participants with BP and 2938 controls from the WTCCC- 1 study, and an additional 2725-independent controls from the WTCCC-2 study. Clinical assessment included a semi-structured interview and review of case notes. Ratings of symptom occurrence and course of illness were made using the Bipolar Affective Disorder Dimension Scale ${ }^{19}$ and/or the operational criteria (OPCRIT) item check list. ${ }^{20}$ Diagnoses were based on all available data using the Research Diagnostic Criteria. ${ }^{21}$ Both WTCCC-1 and WTCCC-2 controls were evenly ascertained from the 1958 British Birth Cohort and the UK Blood Service Control Group samples.

German sample. The collection and genotyping of the German BP sample has been recently described. ${ }^{22}$ In brief, probands with bipolar I disorder were recruited through consecutive hospital admissions and assessed using a structured interview and the OPCRIT checklist. Bestestimate diagnoses were assigned according to DSM-IV criteria. A population-based control sample screened for psychiatric disorders was assembled from the PopGen, KORA and Heinz Nixdorf Recall studies.

Mood-incongruent psychosis phenotype. Considerable attention was given to ensuring comparability between the phenotypes across the three samples. While the $\mathrm{NIMH}$, WTCCC and German samples used different diagnostic instruments (that is, DIGS, Bipolar Affective Disorder Dimension Scale, OPCRIT) to diagnose participants, the psychotic symptoms queried by these interviews are quite similar and, in many cases, identical. In all studies, moodincongruence was defined according to the DSM-IV definition as psychotic "content... inconsistent with depressive themes such as guilt, illness, personal inadequacy or catastrophe... [or] inconsistent with manic themes such as inflated worth, power, knowledge, identity, or special relationship to a deity or famous person.' Further DSM-IV mood-incongruent psychotic symptoms include 'delusions of thought insertion .... of thought broadcasting, and... of control'.

For the NIMH samples, the designation of psychotic features was based on a lifetime history of auditory or visual hallucinations or delusions. In the DIGS subjects are asked about the presence of psychotic symptoms during their most severe depression, as well as during a subsequent review of lifetime psychotic symptoms. Interviewers were asked to determine the incongruence of psychotic content following descriptions of hallucinations or delusions in a mood episode. At least one positive response to these questions in either depressive or manic episodes rendered a subject positive for a lifetime history of mood-incongruence. If insufficient information was available to rule in or rule out a lifetime presence of mood-incongruent psychosis, the case was designated as indeterminate. In the genotyped NIMH sample of $2191 \mathrm{BP}$ cases, 1372 (62.6\%) were classified as having a lifetime history of psychosis and 960 (43.8\%) met criteria for a lifetime history of mood-incongruent psychosis.

The WTCCC study used a semi-structured diagnostic instrument similar to the DIGS, which was then used to complete the Bipolar Affective Disorder Dimension Scale and OPCRIT rating scales. The designation of mood-incongruence was made if a case had a rating of $>20$ in the Bipolar Affective Disorder Dimension Scale mood-incongruent dimension or if the OPCRIT ratings (questions 54, 58, 59, 61-63, $66-68,72-77$ ) were positive for any of the DSM-IV moodincongruent items. Out of the 1868 cases from the WTCCC-1 study, 873 (46.7\%) were classified as having had MICP.

In the German study, the designation of mood-incongruence was made using the OPCRIT items that reflected the DSM-IV definition. Of the 645 cases with BPI, 363 (56.3\%) were classified as having had mood-incongruent psychosis.

\section{Genotyping}

National Institute of Mental Health Bipolar Disorder Consortium. Unrelated cases and controls were genotyped in two separate stages by the Bipolar Genome Study. In all, 1001 cases and 1034 controls selected for the Genetic Association Information Network study were genotyped by 
the Broad Institute using the Affymetrix 6.0 platform (Santa Clara, CA, USA), while an additional 1190 cases and 401 controls were genotyped by the Translational Genomics Research Institute, also on the Affymetrix 6.0 platform. We included the final cleaned data set from the primary casecontrol analysis of each sample. The Genetic Association Information Network-BP sample included genotype data on 724067 single-nucleotide polymorphisms (SNPs). The Translational Genomics Research Institute data set included genotype data on 728187 SNPs.

Wellcome Trust Case-Control Consortium. Genotyping was conducted in two stages, with cases and controls available from WTCCC-1 and controls only from WTCCC-2. The combined WTCCC-1 and 2 samples consisted of 1868 cases and 5682 controls (2957 from WTCCC-1 and 2725 from WTCCC-2). There were 382370 SNPs generated using Affymetrix 5.0 from stage 1 and 696889 SNPs generated from the Affymetrix 6.0 platform in stage 2 .

German sample. The genotyping of the German BP sample has been recently described. ${ }^{22}$ Genotyping was performed on the Illumina HumanHap550 array (Illumina Inc, San Diego, CA, USA). We utilized the final cleaned data set, which included 645 cases and 1310 controls with genotype data on 516024 SNPs.

\section{Data management}

Quality control. Each data set underwent identical quality control steps. We dropped subjects with a missing data rate $\geqslant 3 \%$, and SNPs with a missing data rate $\geqslant 5 \%$. SNPs were also dropped if their frequency was $<1 \%$ or if they had a Hardy-Weinberg equilibrium $P<0.001$. The quality control steps were applied to each study individually before and after imputation, as well as to the combined mega-analysis sample. To assess for cryptic relatedness, genotyped SNPs for each study were combined and identity by descent analysis was performed using a linkage disequilibriumpruned data set. We removed one of any pair of individuals with identity by descent score sharing $>0.2$.

Principal component analysis. To account for population stratification in the GWAS sample, we performed principal component analysis within each data set using markers in linkage equilibrium $\left(R^{2}<0.2\right)$ with a minor allele frequency $>5 \%$. We also excluded all AT/GC SNPs and all SNPs within the major histocompatibility complex (chr6: 25-23 Mb) and chromosome 8 inversion (chr8: 7-13 Mb) regions.

Principal components were selected based on visualization of a screen plot (graphs of the principal components are shown in Supplementary Figure 1). Plotting of the principal components showed two, 11, and one clear outlier(s) in the NIMH, WTCCC and German data sets, respectively, that were removed.

Imputation. Imputation was performed to facilitate a metaanalysis of the three data sets, which were originally genotyped on Affymetrix 5.0 (WTCCC-1), Affymetrix 6.0 (all NIMH and the WTCCC-2 control samples) and Illumina HumanHap550v3 microarray chips. We used BEAGLE to flip

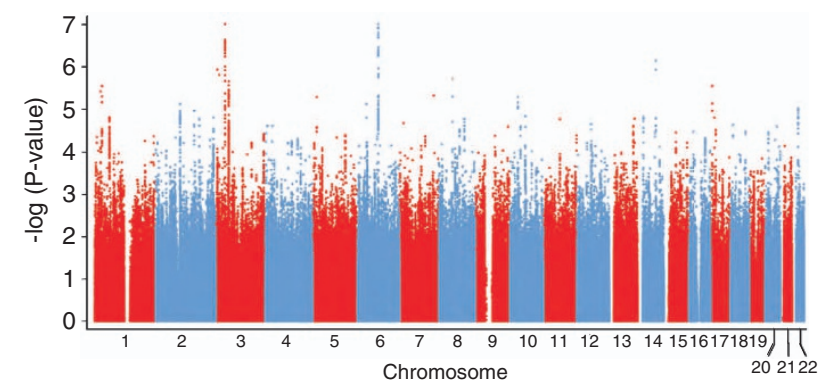

Figure 1 Manhattan plot of the meta-analysis comparing cases with bipolar disorder with mood-incongruent psychotic features to normal controls.

orientation to the positive strand and to impute allelic dosages for autosomal SNPs in the cases and controls. Each data set was imputed separately using phased haplotype data from HapMap I \& II release 24 (http:// hapmap.ncbi.nlm.nih.gov/) as the reference panel. The WTCCC, NIMH, and German samples were imputed separately and subsequently combined. Quality control filters were applied to the individual studies and to the combined data set. SNPs were removed according to the following criteria: minor allele frequency $<0.01, R^{2}<0.3$, Hardy-Weinberg equilibrium $P<0.001$ and a PLINK INFO score $<0.8$. The final imputed files contained 2488722 (NIMH), 2404996 (WTCCC) and 2472086 (German) markers.

\section{Data analysis}

Association analysis. We used the imputed allelic data from the NIMH, WTCCC and German data sets and performed test of association in logistic regression analysis for each dataset, comparing cases with MICP versus controls. Tests of association were performed in PLINK using allelic dosages in an additive logistic regression model including covariates for the top principal components. As a measure of potential residual population stratification, we used WGAviewer (Duke University, Durham, NC, USA) to calculate lambda $(\lambda)$ as the ratio of the median observed versus expected $\chi^{2}$ statistic. To facilitate comparison with other samples, we also calculated a standardized lambda $\left(\lambda_{1000}\right)$ for a sample of 1000 cases and 1000 controls.

Case-only analysis. We performed a case-only analysis of our top findings. Principal components and association studies were performed in each individual sample using all cases with MICP versus all other cases without MICP. As several variables can lead to the designation of MICP, we included as negative only those subjects without any missing data for those variables.

Meta-analysis. Association analysis of each data set was performed with data set-specific principal components. Metaanalysis was subsequently performed in PLINK under a fixed effect model for both the case-control and case-only analysis.

Polygenic analysis. Using the PGC SZ results as a training set, we performed a polygenic analysis of each of the three samples in cases with and without MICP. The PGC SZ 
Table 1 Meta-analysis results for the comparison of bipolar disorder cases with mood-incongruent psychotic features to normal controls, showing markers associated with a $P<1 \times 10^{-5}$

\begin{tabular}{|c|c|c|c|c|c|c|c|c|}
\hline Chromosome & Location (bp) & Marker & $\begin{array}{c}\text { Relation to } \\
\text { gene }\end{array}$ & $\begin{array}{c}\text { Distance to } \\
\text { gene (bp) }\end{array}$ & Gene & $\begin{array}{l}\text { Risk } \\
\text { allele }\end{array}$ & $\begin{array}{l}\text { Odds } \\
\text { ratio }\end{array}$ & $\mathrm{P}$ (meta) \\
\hline 6 & 84283314 & rs1171113 & Intronic & 0 & PRSS35/SNAP91 & C & 1.23 & $9.67 \times 10^{-8}$ \\
\hline 3 & 36831034 & rs9834970 & Upstream & 12278 & TRANK1/LBA & $\mathrm{T}$ & 0.82 & $9.71 \times 10^{-8}$ \\
\hline 14 & 72836967 & rs2333194 & Intronic & 0 & NUMB & $A$ & 0.83 & $7.03 \times 10^{-7}$ \\
\hline 3 & 5680052 & rs11710433 & Intergenic & & N/A & $\mathrm{C}$ & 0.81 & $1.16 \times 10^{-6}$ \\
\hline 3 & 13712001 & rs4450798 & Intergenic & 57078 & FBLN2 & $\mathrm{T}$ & 1.26 & $1.54 \times 10^{-6}$ \\
\hline 8 & 54717753 & rs11773966 & Intronic & 0 & ATP6V1H & $A$ & 2.17 & $1.92 \times 10^{-6}$ \\
\hline 3 & 52693320 & rs10865974 & Intronic & 0 & PBRM1/GNL3 & $\mathrm{T}$ & 0.84 & $2.18 \times 10^{-6}$ \\
\hline 17 & 6034675 & rs3744728 & Intergenic & 66204 & WSC domain containing 1 & C & 1.51 & $2.80 \times 10^{-6}$ \\
\hline 1 & 30298301 & rs2860031 & Intergenic & & N/A & $\mathrm{G}$ & 0.82 & $2.81 \times 10^{-6}$ \\
\hline 1 & 24304543 & rs3934861 & Intronic & 0 & MYOMЗ & A & 0.8 & $3.76 \times 10^{-6}$ \\
\hline 7 & 137509306 & rs10255295 & Intergenic & 55716 & $A K R 1 D 1$ & G & 1.31 & $4.73 \times 10^{-6}$ \\
\hline 10 & 32349011 & rs1775715 & Intronic & 0 & KIF5B & $\mathrm{G}$ & 1.18 & $5.13 \times 10^{-6}$ \\
\hline 5 & 17268444 & rs2962370 & Upstream & 2306 & $B A S P 1$ & $\mathrm{G}$ & 1.19 & $5.14 \times 10^{-6}$ \\
\hline 6 & 37282723 & rs1680005 & Upstream & 5209 & TMEM217 & A & 1.18 & $7.45 \times 10^{-6}$ \\
\hline 2 & 98450324 & rs12617721 & Intronic & 0 & INPP4A & C & 1.2 & $7.45 \times 10^{-6}$ \\
\hline 22 & 24205265 & rs1930961 & Intergenic & 17620 & $C R Y B B 2 P 1$ & $\mathrm{C}$ & 0.72 & $9.49 \times 10^{-6}$ \\
\hline
\end{tabular}

a

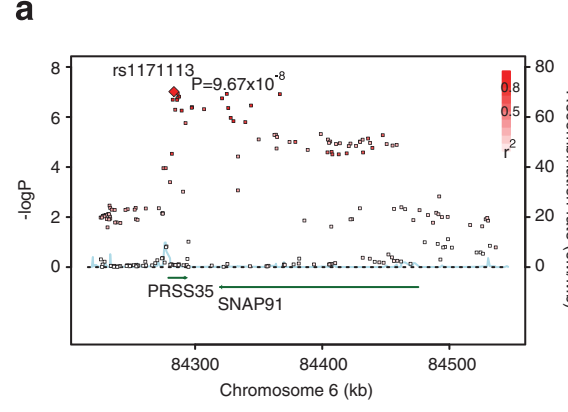

rs1171113
OR $(95 \% \mathrm{Cl})$

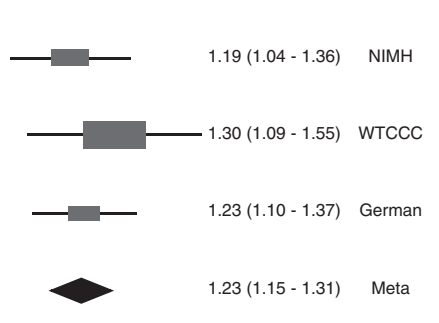

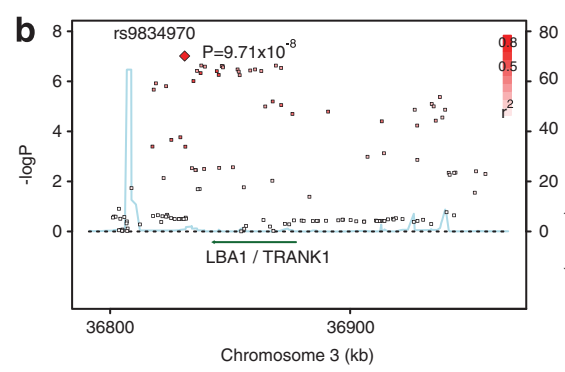

rs9834970
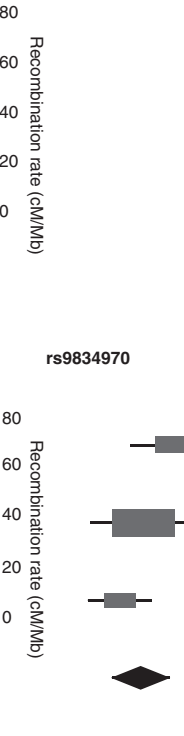

rs2333194
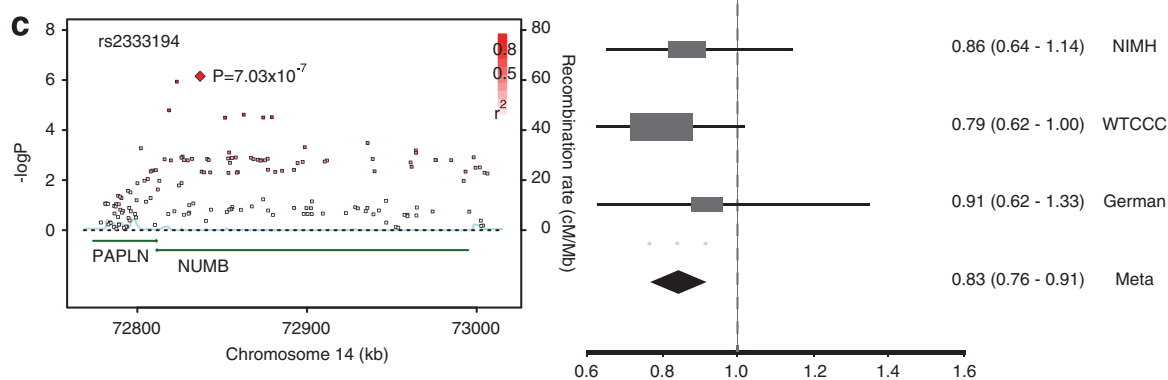

Figure 2 Regional and forest plots of the top three findings in the case-control meta-analysis. Results are shown for: (a) PRSS35/SNAP91, (b) TRANK1/LBA1 and (c) NUMB. Genome coordinates are based on NCBI 36/hg 18 build and recombination rates are derived from the HapMapll CEU sample.

results were clumped into a data set of 85284 markers in linkage equilibrium $\left(r^{2}<0.25\right)$ using the CEU HapMapll samples as a reference. We used PLINK to calculate polygenic scores using the SZ risk alleles weighted by their $\log (\mathrm{OR})$ as the initial training set sample. Polygenic scores were derived in each individual MICP samples using several 
Table 2 Association of PGC schizophrenia polygenic scores with moodincongruent psychosis in cases with bipolar disorder

\begin{tabular}{lcc}
\hline $\begin{array}{l}\text { Threshold P-value } \\
\text { (PGC schizophrenia } \\
\text { training data set) }\end{array}$ & $\begin{array}{c}\text { Regression } \\
\text { Z-statistic }\end{array}$ & $\begin{array}{c}\text { MICP case-only } \\
\text { P-value }\end{array}$ \\
\hline$P<0.05$ & & 0.01 \\
$P<0.1$ & 2.59 & 0.01 \\
$P<0.2$ & 2.59 & 0.003 \\
$P<0.3$ & 3.00 & 0.004 \\
$P<0.4$ & 2.91 & 0.004 \\
$P<0.5$ & 2.86 & 0.006 \\
\hline
\end{tabular}

Abbreviations: MICP, mood-incongruent psychotic features; PGC, Psychiatric GWAS Consortium.

$P$-value thresholds. A case-only logistic regression was subsequently performed for each data set using the polygenic scores as the dependent variable and each study's principal components as covariates. Fixed effect metaanalysis of each association was subsequently performed in STATA (Stata Corp, College Station, TX, USA) using its 'meta' function. As a majority of the controls in the MICP analysis (5022 out of 8148 ) overlapped with the controls in the PGC schizophrenia (SZ) analysis, we restricted our MICP analysis to a case-only analysis.

\section{Results}

We first performed association analyses of each individual data set. The number of cases with mood-incongruent psychosis across the three data sets was 960 (43.8\%) in the NIMH, 873 (46.7\%) in the WTCCC and 363 (56.3\%) in the German samples. Quantile-quantile (QQ) plots for each sample are shown in Supplementary Figure 1 . The $\lambda$ estimates were 1.01, 1.04 and 1.02 for the NIMH, WTCCC and German samples, respectively. There were no genomewide significant findings within each sample, leading us to proceed to a fixed effect meta-analysis of the three data sets. The meta-analysis included a total of 2196 cases with BP with MICP and 8418 controls.

As shown in Figure 1 and Table 1, the most highly associated marker in the meta-analysis was rs1171113, with the $\mathrm{C}$ allele being overrepresented in cases (odds ratio $\left.(\mathrm{OR})=1.23, \quad P=9.67 \times 10^{-8}\right)$. Figure $2 \mathrm{a}$ highlights this association in more detail, showing that rs1171113 tags an $\sim 150-200 \mathrm{~kb}$ region of chromosome $6 q 14.2$ that encompasses the genes PRSS35 (protease, serine 35) and SNAP91 (synaptosomal-associated protein, $91 \mathrm{kDa}$ homolog, also known as clathrin coat assembly protein AP180). The second strongest association was found on chromosome 3p22.2 with the rs9834970 marker (risk allele $=\mathrm{T}$; OR $=0.82$; $P=9.71 \times 10^{-8}$ ). As shown in Figure $2 \mathrm{~b}$, this marker is $\sim 15 \mathrm{~kb}$ downstream of TRANK1 (tetratricopeptide repeat and ankyrin repeat containing 1 gene), also known as $L B A 1$ (lupus brain antigen 1). Finally, Figure $2 \mathrm{c}$ shows the third strongest finding with a $P<10^{-6}$, on chromosome 14q24.2 (rs2333194; $P=7.0 \times 10^{-7}$ ), which is in the third intron of NUMB. To determine whether these top findings were specifically associated with MICP, we performed a logistic regression analysis comparing $\mathrm{BP}$ cases with $\mathrm{MICP}$ versus all other BP cases. We found evidence of association of the risk alleles with MICP cases in two of our top findings: rs1171113 $(\mathrm{OR}=1.14, P=0.014)$ and $\mathrm{rs} 2333194(\mathrm{OR}=1.12, P=0.02)$, but not in rs9834970 (OR $=1.04, P=0.42)$.

To test the hypothesis that the MICP subset of BP may be associated with SZ, we performed a polygenic analysis of the MICP data set using a case-only design, as most of the controls in this study overlap with those of the PGC SZ study. First, we selected common markers between the PGC SZ and our MICP data sets and subsequently performed linkage disequilibrium-pruning to obtain markers in approximate linkage disequilibrium $\left(r^{2}<0.25\right)$. We derived a polygenic score from the PGC SZ analysis using several association $P$ value thresholds, ranging from $0.05-0.5$, and performed a logistic regression analysis in each case-only sample, testing whether an individual's polygenic score was associated with the presence or absence of MICP. Each sample was corrected for by its study-specific principal components. In a meta-analysis of the three samples, we found significant evidence for polygenic overrepresentation of SZ risk alleles in BP cases with MICP (Table 2), with the strongest evidence of association found at the $P<0.2$ threshold $\left(P_{\text {meta }}=0.003\right)$. Evidence of association was found across all $P$-value thresholds, suggesting that this association was unlikely to be spurious.

\section{Discussion}

In this study we have combined three large BP GWAS samples to test the hypotheses that (1) a subphenotype of BP, marked by MICP, would show association in loci not found in analyses of the more broadly defined phenotype; and (2) that the MICP BP subphenotype would show evidence at a broader level for a relationship with SZ. Our meta-analysis of 2196 cases and 8418 controls found association of several markers with $P<1 \times 10^{-6}$, though none reached genomewide significance. Prior family studies suggested that mood disorders with MICP might be etiologically related to SZ. ${ }^{14,23}$ In support of this hypothesis, our polygenic analysis using risk alleles from the PGC SZ GWAS study found an association, en masse, between large numbers of $S Z$ variants and the presence of MICP in BP cases, suggesting that genetic overlap across syndromes is potentially due to shared polygenic variation with individual effects that are likely too small to be detected in single marker GWAS analyses.

The marker with the strongest association (rs1171113), which was enriched in the case-only analysis, resides in a region of high linkage disequilibrium that includes the genes PRSS35 and SNAP91. The PRSS35 gene encodes an inactive serine protease homolog of no known function (reference uniprot database ${ }^{24}$ ). SNAP91 codes for a $91 \mathrm{kDa}$ synaptosomal-associated protein, also known as clathrin assembly protein 180 (AP180), which is enriched in the presynaptic terminal and expressed only in neurons. ${ }^{25}$ SNAP91 is essential for the formation and function of clathrin coated vesicles, which are the major means of recycling vesicles at the presynaptic membrane. ${ }^{26}$ Interestingly, SNAP91 has also been proposed to have a role in calcium signaling and the Wnt pathway. Studies have found that the SNAP91 protein can inhibit two important intracellular signaling molecules, phospholipase $\mathrm{C}-\gamma 1$ and phospholipase 
D1. ${ }^{27,28}$ Both molecules have been implicated in the Wnt pathway $^{29}$ and phospholipase $\mathrm{C}-\gamma 1$ has a pivotal role in intracellular inositol signaling, which is hypothesized to have a role in lithium's mechanism of action. As some studies have suggested that BP with mood-incongruent psychosis is less responsive to lithium treatment, ${ }^{12,30}$ it is intriguing to speculate that SNAP91, via its effect on phospholipase C- $\gamma 1$, may be involved in lithium response.

Our second strongest association signal (rs9834970) is $\approx 12 \mathrm{~kb}$ downstream of the $L B A 1$ gene, which is also known as TRANK1 (tetratricopeptide repeat and ankyrin repeat containing 1), and was originally identified as a brain-specific antigen in a murine model of systemic lupus erythematosus, a human disorder with frequent neuropsychiatric symptoms. ${ }^{31}$ This marker was recently found to have genome-wide significant evidence for association with BP in a large metaanalysis of mixed ancestry samples. ${ }^{32}$ Notably, this marker also showed suggestive evidence for association with $\mathrm{SZ}$ in the PGC SZ meta-analysis $\left(p=3.6 \times 10^{-5}\right) .{ }^{33}$ Our association with this marker was found with far smaller sample sizes; however, we did not find evidence that the rs9834970 risk allele was specifically associated with MICP in a case-only analysis, suggesting that this marker is likely to be representative of a risk allele for the broader diagnosis of BP. Our third strongest association (rs2333194) did show modest overrepresentation in MICP in our case-only analysis and was found in $N U M B$, an important modulator of the notch signaling pathway, which among other functions, has been shown to regulate neuronal differentiation in vitro. ${ }^{34}$

Psychiatric phenotypes are likely amalgams of multiple genetic, development and environmental influences. Although single gene findings can illuminate important pathophysiological mechanisms, phenotypic considerations are likely to be more correlated with markers of aggregate genetic effects, such as familiality or 'global' genetic analysis like polygenic risk profiling. ${ }^{35}$ In this study such a polygenic analysis has found evidence for association of the MICP BP phenotype with SZ, which is consistent with prior family studies, and provides further support for etiological ties between the major mood and psychotic disorders. A prior study found a similar association between $S Z$ and a phenotype that is closely related to BP with MICP, that of schizoaffective disorder as diagnosed by the Research Diagnostic Criteria. ${ }^{36}$ Although cases with RDC schizoaffective disorder are included within our diagnosis of BP with MICP, we were not able to derive this RDC-based diagnosis from the DSM-based DIGS interview.

The results of this study should be interpreted in light of several limitations. Perhaps most important is the limited power to detect risk variants of small effect sizes, particularly as loss of statistical power is inherent in the subphenotype approach, which splits a broader case sample into smaller units. Even with the three large BP samples, our study (assuming a common and fully informative marker allele) only had sufficient power to detect ORs in the $\approx 1.3$ range, which is slightly greater than that of our top finding $(O R=1.23)$. Although larger samples will provide increased statistical power, they will also create the challenge that arises from the need to harmonize subphenotype definitions. As patients with psychosis may fail to recall specific psychotic symptoms, ${ }^{37}$ differential misclassification of mood-incongruence is an important concern, particularly in case-only analysis, which prompted us to focus our primary analysis on a case-control design.

In summary, we have performed a large GWAS of the MICP subphenotype and found no associations meeting genomewide significance criteria, although several associations at the 'suggestive' level warrant further investigation. More globally, our polygenic analysis has found modest evidence for association of the MICP BP phenotype with SZ, which is consistent with prior family studies, and provides further support for etiological ties between the major mood and psychotic disorders.

\section{Conflict of interest}

The authors declare no conflict of interest.

Acknowledgements. Supported by NARSAD, NIMH (K99/R00MH086049) and the Wellcome Trust (award 076113). We express our appreciation to the families and individuals who participated in this project. We thank those involved in the BP Research Network (bdrn.org) and the MDF-The Bipolar Organization for the help of its staff and members. Funding for recruitment and phenotype assessment has been provided by the Wellcome Trust and the Medical Research Council. This study makes use of data generated by the Wellcome Trust Case-Control Consortium. A full list of the investigators who contributed to the generation of the data is available from http://www.wtccc.org.uk. The NIMH samples were genotyped under the direction of Bipolar Genome Study. The Principal Investigators and Co-Investigators were: John R Kelsoe, Tiffany A Greenwood, Caroline M Nievergelt, Rebecca McKinney, Paul D Shilling, Nicholas Schork, Erin N Smith, Cinnamon Bloss, John Nurnberger, Howard J Edenberg, Tatiana Foroud, Daniel L Koller, Elliot Gershon, Chunyu Liu, Judith A Badner, William A Scheftner, William B Lawson, Evaristus A Nwulia, Maria Hipolito, William Coryell, John Rice, William Byerley, Francis McMahon, Thomas G Schulze, David T Chen, Wade Berrettini, James B Potash, Peter P Zandi, Pamela B Mahon, Melvin G Mclnnis, Sebastian Zöllner, Peng Zhang, David W Craig, Szabolcs Szelinger, Thomas B Barrett.

1. Bromet EJ, Kotov R, Fochtmann LJ, Carlson GA, Tanenberg-Karant M, Ruggero C et al. Diagnostic shifts during the decade following first admission for psychosis. Am J Psychiatry 2011; 168: 1186-1194.

2. Kieseppa T, Partonen T, Haukka J, Kaprio J, Lonnqvist J. High concordance of bipolar I disorder in a nationwide sample of twins. Am J Psychiatry 2004; 161: 1814-1821.

3. Sklar P, Ripke S, Scott LJ, Andreassen OA, Cichon S et al., Psychiatric GWAS Consortium Bipolar Disorder Working Group. Large-scale genome-wide association analysis of bipolar disorder identifies a new susceptibility locus near ODZ4. Nat Genet 2011; 43: 977-983.

4. McClellan J, King MC. Genetic heterogeneity in human disease. Cell 2010; 141: 210-217.

5. Purcell SM, Wray NR, Stone JL, Visscher PM, O'Donovan MC et al., International Schizophrenia Consortium. Common polygenic variation contributes to risk of schizophrenia and bipolar disorder. Nature 2009; 460: 748-752.

6. Angst J. Psychiatric diagnoses: the weak component of modern research. World Psychiatry 2007; 6: 94-95.

7. Jaspers K. General Psychopathology. The Johns Hopkins University Press: Baltimore, USA, 1997.

8. Toni C, Perugi G, Mata B, Madaro D, Maremmani I, Akiskal HS. Is mood-incongruent manic psychosis a distinct subtype? Eur Arch Psychiatry Clin Neurosci 2001; 251: 12-17.

9. Goes FS, Zandi PP, Miao K, McMahon FJ, Steele J, Willour VL et al. Mood-incongruent psychotic features in bipolar disorder: familial aggregation and suggestive linkage to $2 \mathrm{p} 11$ q14 and 13q21-33. Am J Psychiatry 2007; 164: 236-247.

10. Tohen M, Tsuang MT, Goodwin DC. Prediction of outcome in mania by mood-congruent or mood-incongruent psychotic features. Am J Psychiatry 1992; 149: 1580-1584.

11. Harrow M, Grossman LS, Herbener ES, Davies EW. Ten-year outcome: patients with schizoaffective disorders, schizophrenia, affective disorders and mood-incongruent psychotic symptoms. Br J Psychiatry 2000; 177: 421-426.

12. Maj M, Pirozzi R, Bartoli L, Magliano L. Long-term outcome of lithium prophylaxis in bipolar disorder with mood-incongruent psychotic features: a prospective study. J Affect Disord 2002; 71: 195-198.

13. Coryell W, Tsuang MT. Major depression with mood-congruent or mood-incongruent psychotic features: outcome after 40 years. Am J Psychiatry 1985; 142: 479-482. 
14. Maier W, Lichtermann D, Minges J, Heun R, Hallmayer J, Benkert O. Schizoaffective disorder and affective disorders with mood-incongruent psychotic features: keep separate or combine? evidence from a family study. Am J Psychiatry 1992; 149: 1666-1673.

15. Kendler KS, Karkowski-Shuman L, Walsh D. The risk for psychiatric illness in siblings of schizophrenics: the impact of psychotic and non-psychotic affective illness and alcoholism in parents. Acta Psychiatr Scand 1996; 94: 49-55.

16. Hamshere ML, Schulze TG, Schumacher J, Corvin A, Owen MJ, Jamra RA et al. Moodincongruent psychosis in bipolar disorder: conditional linkage analysis shows genome-wide suggestive linkage at 1q32.3, 7p13 and 20q13.31. Bipolar Disord 2009; 11: 610-620.

17. Smith EN, Bloss CS, Badner JA, Barrett T, Belmonte PL, Berrettini W et al. Genome-wide association study of bipolar disorder in European American and African American individuals. Mol Psychiatry 2009; 14: 755-763.

18. Sanders AR, Levinson DF, Duan J, Dennis JM, Li R, Kendler KS et al. The internetbased MGS2 control sample: self report of mental illness. Am J Psychiatry 2010; 167 854-865.

19. Craddock N, Jones I, Kirov G, Jones L. The bipolar affective disorder dimension scale (BADDS)-a dimensional scale for rating lifetime psychopathology in bipolar spectrum disorders. BMC Psychiatry 2004; 4: 19.

20. McGuffin P, Farmer A, Harvey I. A polydiagnostic application of operational criteria in studies of psychotic illness. Development and reliability of the OPCRIT system. Arch Gen Psychiatry 1991; 48: 764-770.

21. Spitzer RL, Endicott J, Robins E. Research diagnostic criteria: rationale and reliability. Arch Gen Psychiatry 1978; 35: 773-782.

22. Cichon S, Muhleisen TW, Degenhardt FA, Mattheisen M, Miro X, Strohmaier $\mathrm{J}$ et al. Genome-wide association study identifies genetic variation in neurocan as a susceptibility factor for bipolar disorder. Am J Hum Genet 2011; 88: 372-381.

23. Kendler KS, McGuire M, Gruenberg AM, O'Hare A, Spellman M, Walsh D. The roscommon family study. IV. affective illness, anxiety disorders, and alcoholism in relatives. Arch Gen Psychiatry 1993; 50: 952-960.

24. Healy DG, Falchi M, O'Sullivan SS, Bonifati V, Durr A, Bressman S et al. Phenotype, genotype, and worldwide genetic penetrance of LRRK2-associated parkinson's disease: a case-control study. Lancet Neurol 2008; 7: 583-590.

25. Yao PJ, Coleman PD, Calkins DJ. High-resolution localization of clathrin assembly protein AP180 in the presynaptic terminals of mammalian neurons. J Comp Neurol 2002; 447 $152-162$.

26. Ryan TA. A pre-synaptic to-do list for coupling exocytosis to endocytosis. Curr Opin Cell Biol 2006; 18: 416-421.
27. Han SJ, Lee JH, Hong SH, Park SD, Kim CG, Song MD et al. AP180 binds to the C-terminal SH2 domain of phospholipase C-gamma1 and inhibits its enzymatic activity. Biochem Biophys Res Commun 2002; 290: 35-41.

28. Cho JH, Oh DY, Kim HJ, Park SY, Choi HJ, Kwon SJ et al. The TSP motif in AP180 inhibits phospholipase D1 activity resulting in increased efficacy of anticancer drug via its direct binding to carboxyl terminal of phospholipase D1. Cancer Lett 2011; 302: 144-154.

29. Kohn AD, Moon RT. Wht and calcium signaling: Beta-catenin-independent pathways. Cell Calcium 2005; 38: 439-446.

30. Pfennig A, Schlattmann P, Alda M, Grof P, Glenn T, Muller-Oerlinghausen B et al. Influence of atypical features on the quality of prophylactic effectiveness of long-term lithium treatment in bipolar disorders. Bipolar Disord 2010; 12: 390-396.

31. Moore PM, Vo T, Carlock LR. Identification and cloning of a brain autoantigen in neurobehavioral SLE. J Neuroimmunol 1998; 82: 116-125.

32. Chen DT, Jiang X, Akula N, Shugart YY, Wendland JR, Steele CJ et al. Genome-wide association study meta-analysis of european and asian-ancestry samples identifies three novel loci associated with bipolar disorder. Mol Psychiatry advance online publication, 20 December 2011; doi:10.1038/mp.2011.157 (e-pub ahead of print).

33. Ripke S, Sanders AR, Kendler KS, Levinson DF, Sklar P, Holmans PA et al. Genome-wide association study identifies five new schizophrenia loci. Nat Genet 2011; 43: 969-976.

34. Sestan N, Artavanis-Tsakonas S, Rakic P. Contact-dependent inhibition of cortical neurite growth mediated by notch signaling. Science 1999; 286: 741-746.

35. Kendler KS. The dappled nature of causes of psychiatric illness: replacing the organicfunctional/hardware-software dichotomy with empirically based pluralism. Mol Psychiatry 2012; 17: 377-388.

36. Hamshere ML, O'Donovan MC, Jones IR, Jones L, Kirov G, Green EK et al. Polygenic dissection of the bipolar phenotype. Br J Psychiatry 2011; 198: 284-288

37. Pulver AE, Carpenter WT Jr. Lifetime psychotic symptoms assessed with the DIS. Schizophr Bull 1983; 9: 377-382.

Translational Psychiatry is an open-access journal published by Nature Publishing Group. This work is licensed under the Creative Commons Attribution-NonCommercialShare Alike 3.0 Unported License. To view a copy of this license, visit http://creativecommons.org/licenses/by-nc-sa/3.0/

Supplementary Information accompanies the paper on the Translational Psychiatry website (http://www.nature.com/tp) 